\title{
CT and MR Compatible Light Puncture Robot: Architectural Design and First Experiments
}

\author{
Elise Taillant ${ }^{1,2}$, Juan-Carlos Avila-Vilchis ${ }^{1,3}$, Christophe Allegrini ${ }^{1}$, \\ Ivan Bricault ${ }^{1,4}$, and Philippe Cinquin ${ }^{1}$ \\ 1 Laboratoire TIMC-IMAG, Equipe GMCAO, 38706 La Tronche CEDEX, France, \\ [elise.taillant,philippe.cinquin]@imag.fr \\ 2 PRAXIM Medivision SA, 38700 La Tronche, France \\ 3 Universidad Autónoma del Estado de México 50130, Toluca, Mexico \\ ${ }^{4}$ Service Central d'Imagerie Médicale CHU Grenoble. 38700 La Tronche, France ${ }^{\ddagger}$
}

\begin{abstract}
This paper presents a new robotic architecture designed to perform interventional CT/MR procedures, particularly punctures. Such procedures are very popular nowadays for diagnostic or therapeutic purposes. Innovations concerning the robotic architecture, materials and energy sources are exposed. We also introduce the control loop we use to check the movements and the positioning of the robot, including a new method to localize the robot thanks to the images coming from the imaging devices (CT or MRI). Finally, the results of the first experiments are presented.
\end{abstract}

\section{Introduction}

$\mathrm{CT} / \mathrm{MR}$ image-guided interventional procedures are becoming more and more popular, either for diagnostic or therapeutic purposes. Many types of imageguided biopsies are performed routinely, and new percutaneous techniques such as radiofrequency treatment allow efficient tumor ablations with reduced trauma and short recovery time. Nevertheless, even with MR or CT image guidance, the precise insertion of biopsy needles or tumor ablation applicators remains a challenging task. Since it is difficult for physicians to reproduce accurately an oblique $3 \mathrm{D}$ path, they often restrict needle trajectories to vertical insertions parallel to the image plane. This can limit the possibility of targeting a lesion while avoiding critical structures. Furthermore, physicians usually do not benefit from a real-time imaging feedback during the procedure, either because they want to avoid CT radiation exposure when they manipulate the needle, or because realtime imaging is not available. As a consequence, the needle insertion procedure requires that physicians switch many times between the patient and the image display room, as they achieve a succession of small needle movements and control imaging acquisitions. This causes an important loss of time. Moreover, without real-time imaging control, it is difficult to adapt the trajectory to soft tissue and

\footnotetext{
$¥$ We would like to thank the Radiology Department (Pr. Coulomb) and the Magnetic Resonance Unit (Pr. Le Bas) of Grenoble's University Hospital for their kind assistance in the experiments.
} 
organ deformations along the needle path. Finally, a precise achievement of the planned trajectory often relies on multiple trials and needle repositioning that may cause more trauma than expected to the patient. Since a few years, systems and methods have been thought up in order to improve needle placement accuracy and to reduce interventions durations as well as radiation exposition. Non-automated methods were invented to help the physicians to perform their task such as the image overlay system with enhanced reality presented in 1 by Masamune et al. or the laser beam guidance for interventional CT by Gangi et al. [2] which allows oblique insertions by aligning the needle with the laser beam. These two methods help physicians to perform a more accurate insertion of the needle but still do not eliminate radiations exposure of the physician.

In the past few years, robots, either guided by physicians with joysticks or autonomous but under physician supervision, have been developped as well as various methods of registration to guide the robot and check its position. In [3], a method for CT guided needle placement is proposed and consists of a localization module (a Brown-Roberts-Well frame) placed on a needle-holding end-effector to localize the effector in the image space using a single CT image. A single cross-section of the frame corresponds to a unique position of the end-effector and allows consequently to know the position of the needle in the body of the patient. This localization method combined with Stoianovici et al. work on a needle driver and a robotic system [4] is used to perform percutaneous interventions [5]. Patriciu et al., in [6], present a robot and needle positioning technique based on laser alignment which does not require any $\mathrm{CT}$ image and thus does not expose the patient to radiations but cannot be used to check needle orientation during interventions. Although these methods are proved to work well, most of them were designed for classical robotic arms which are unwieldy in a scanner room, worrisome for the patient and difficult to implement under MR environments.

Several MR compatible devices used to perform biopsies already exist and work well. ROBITOM [7] is a MR compatible robot which works in the isocenter of a closed high-field MR system but it is dedicated only to breast biopsy and therapy and one of its degrees of freedom is manually controlled. Chinzei et al in [8], present a MR compatible surgical assistance robot which is designed to cooperate with a surgeon and position a tool such as a laser pointer or a biopsy catheter. Some drawbacks of this system are that it works only with an opened MR system which implies a bad image quality and that it is quite cumbersome. A MR compatible manipulator for transrectal prostate biopsy with a remote manual actuation is presented in [10] and 9].

The work presented here focuses on a new CT/MRI compatible robotic architecture (LPR for Light Puncture Robot) interdependent of the patient's body, intrinsically compliant, and designed to perform puncture interventions. We also describe the control loop based on images coming from the imaging system and used to check LPR's position and orientation.

Usually punctures are performed for tumoral pathologies. In these cases, lesions smaller than $1 \mathrm{~cm}$ are hardly characterized and usually followed up rather than treated. Consequently, our goal is to be able to reach targets larger than $1 \mathrm{~cm}$. 


\section{Material and Methods}

\subsection{Robot Architecture}

The LPR possesses 5 DOF (Degree Of Freedom). The platform provides the mechanical support to perform translation thanks to 4 straps bound to the platform on one side and to a support frame on the other side where the actuators for each strap are situated. Translation is performed over the patient's body, which gives a natural orientation to the robot (see fig. 1).

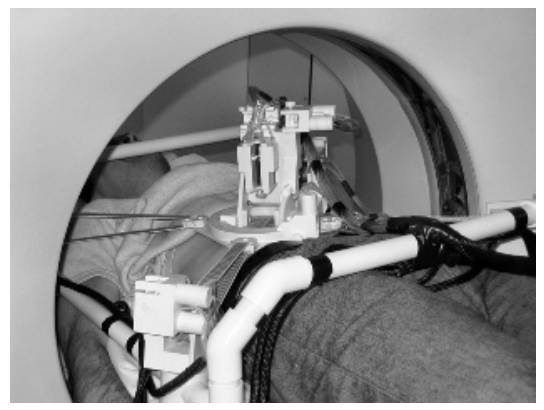

Fig. 1. Robot on Patient Inside CT Gantry

This architecture provides intrinsic compensation of the physiological movements of the patient i.e. the robot follows patient's abdomen movements such as breathing. The robot base can rotate w.r.t. (with respect to) an axis normal to the patient's body thus defining the azimuthal angle $\theta \in[0 ; 2 \pi]$. This rotation is performed in both directions without restrictions. The trocar holder rotates with the base and has a proper rotation (w.r.t the robot's base) defining the polar angle $\phi \in\left[-\frac{\pi}{18} ; \frac{\pi}{3}\right]$. Each motion is performed by a couple of pneumatic actuators powered by compressed air at the pressure of 3.5 bar. Each actuator is composed of a piston which can drag with an associated sprocket wheel (see fig. 21). This movement is possible only in one direction thus permitting to block the robot in a certain position. Each cylinder possesses two compressed air inlets, one on each of its sides. Air is alternatively injected in each compartment and makes the piston move and push the sprocket wheel by one increment thus the movement is very easily and precisely controlled. A worm is assembled to the sprocket wheel axis and works with its corresponding gear. For each movement the opposite direction is achieved by the complementary actuator. For translation, the actuators have a pulley that allows the straps to be entangled/disentangled. A simple pneumatic actuator is used for the trocar clamping task. In order to perforate the skin, another simple actuator allows a $2 \mathrm{~cm}$ fast translation of the trocar.

Plastic materials used construct the LPR are MR compatible and completely transparent under MRI, they do not create artifacts under CT scanner. The robot only weighs $1 \mathrm{~kg}$. The valving system which controls the actuators is linked to them by $7 \mathrm{~m}$ long plastic tubes thus allowing it not to be in the CT/MR room. 


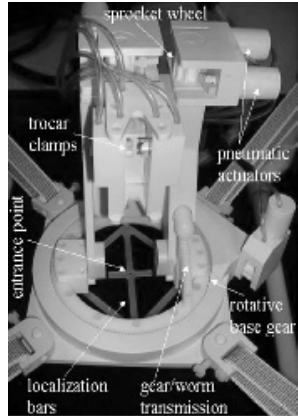

(a)

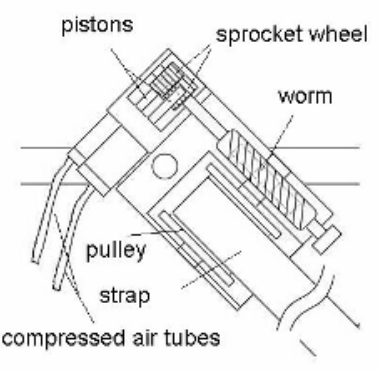

(b)

Fig. 2. (a)Light puncture robot, main part, (b) Schema of actuators

\subsection{Localization Module}

Because of LPR's medical applications under CT/MRI environment, it is not possible to use sensors to control its movements and position. Optical or magnetic localizers are not suitable for MRI and furthermore do not give a real feedback as tissues deformations and organ movements are not taken into account. Images coming from the imaging device (CT or MRI) are the best feedback and are consequently used in our application to close the control loop.

Localization is done thanks to the localization module we designed which is totally integrated into the robot architecture and is composed of two parts. The first one is a $7.5 \mathrm{~cm}$ long and $1.6 \mathrm{~mm}$ thick square frame (and its diagonals), made of epoxy resin charged with fibreglass. This frame is screwed to the rotating base of the robot so that its center is merged with the entry point. A small drilled ball at the center of the frame allows to let the needle go through the frame's diagonals and to position the needle in the correct direction. That frame is used to determine the position of the entry point of the robot as well as the rotation of LPR's rotating base w.r.t the scanner. The second part is a $1.6 \mathrm{~cm} \mathrm{x} 1.6 \mathrm{~cm}$ $\mathrm{x} 6.2 \mathrm{~cm}$ Delrin acetal resin made bar screwed to the needle-holder. It allows to compute the inclination of the needle-holder w.r.t the robot's base and the scanner. For MR applications the same module is used except that all the bars are replaced by small tubes filled with water.

\subsection{Image Based Control of the Robot}

At the beginning of the procedure, a physician chooses the target he wants to reach and the entry point on a first set of images. Setting these two points implies setting the trajectory the needle will follow and so the orientation/position the robot must take to respect the trajectory. When both the trajectory is defined and a first localization of the robot is done, thus giving the initial position, the shortest trajectory from the initial position to the required position is computed. The robot then moves to the choosen position and orientation. When the movement is done, the position/orientation is checked thanks to the images feedback. 
Images are processed and a localization algorithm is applied. If the detected position/orientation is not correct, the robot is moved again and its new position is checked and so on until the desired position is reached.

Image Processing: Depending on the angle between the images and the robot, images will contain either a trace corresponding to the Delrin bar or traces left by the frame (from 2 up to 4 ellipses) or both. As the grey level response of Delrin is different from the grey level response of epoxy, the image processing applied to get the objects depends on the object we want to detect. In both cases, two steps are essential to correctly detect objects. The first step is an estimation of the position of the objects and consists of a hard thresholding followed by morphological operations in order to clear out small objects. We then apply a priori knowledge on the size and on the geometry of the localization device to get the points we are interested in. When the objects' position are approximately found, a second step is performed on the original image to improve the localization in which only small areas around detected points are processed.

Robot Localization: A localization method, using only one slice (as it is done in [3]) which contains both the frame and the Delrin bar, was developed. This method was not used for our application for several reasons. First, using several images improves the precision of the localization. Then, since with MRI, there are no radiations, and since recent multislice CT scanners allow to take several slices at the same time, the acquisition of several images is not a real drawback. Furthermore, it allows the physician to be integrated in the control loop since he is able to see what happens thanks to the multiple slices that were acquired. For these reasons and depending on the position of the robot, we will use a set of images (at least two) to localize it. The only images we can implement are those in which we have detected at least three points for the frame in the image processing step. All the angles and coordinates in this section are to be understood w.r.t the scanner coordinates system, $R_{S}$. The angle $\theta$, corresponding to the rotation of the robot's base w.r.t the scanner, is easily calculated from the equations of the diagonals and the sides of the square frame which are computed from the detected points.

The computation of the coordinates of the intersection of the two diagonals gives the coordinates of the entry point. If one of the equations of the diagonals is not possible to compute (only three points on image see fig. 3.a) then the entry point coordinates are computed as follows :

$$
\begin{gathered}
\left(O_{X}, O_{Y}, O_{Z}\right)=\left(L \times \frac{\sqrt{2}}{2}-d_{2}\right) \times \vec{V}+\left(B_{1_{X}}, B_{1_{Y}}, B_{1_{Z}}\right), \\
\text { with } \left.d_{2}=\min \left(\left\|\overrightarrow{A_{1} B_{1}}\right\|,\left\|\overrightarrow{B_{1} D_{1}}\right\|\right) \times(|\cos (\theta)|+|\sin (\theta)|) \text {, (see fig, [3a }\right) .
\end{gathered}
$$

with $\left(A_{1}, B_{1}, D_{1}\right)$ (respectively $\left(A_{2}, B_{2}, D_{2}\right)$ ) be the intersections between the first image (respectively the second image) and the frame (see fig. [3).

Finally, the inclination $\phi$ of the needle-holder w.r.t $R_{S}$ is calculated. The equation of the line representing the inclination of the needle holder is determined from a set of points belonging both to the localization bar and the needle holder. We then compute the angle between this line and a vertical line. Depending on the 


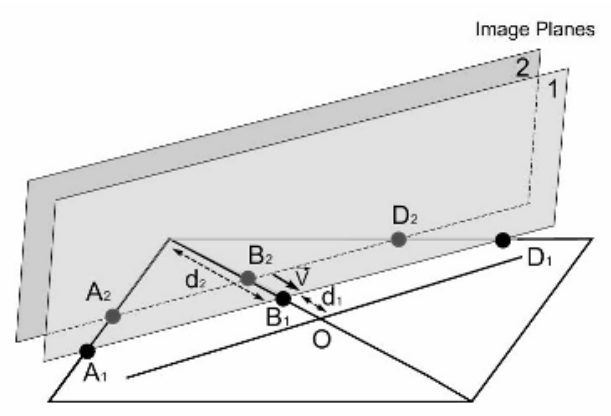

(a)

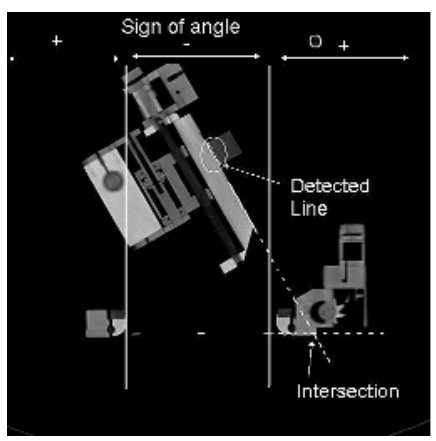

(b)

Fig. 3. (a) Image intersecting the frame in 3 points (b) sign of inclination angle

position of the intersection between this line and the base plane of the robot, we are able to determine if this angle is positive or negative (fig. 3. b).

\section{Experiments and Results}

Open-Loop Performance Experiments: These experiments consist of moving the robot to a particular position/attitude and check its positioning accuracy. They gave the following results assuming that the work surface is a plane. For strap-based translation, accuracy is about $5 \%$ of the displacement $\mathrm{d}$ (if $d=20 \mathrm{~mm}$ then the error is about $1 \mathrm{~mm}$ ). Rotation and inclination accuracy are less than $1^{\circ}$. Repeatability is less than $0.5 \mathrm{~mm}$ for translation and $1^{\circ}$ for rotation.

Image-Based Localization Experiments: Experiments on robot localization with CT images were performed to check accuracy. The middle of the frame corresponding to the point where the needle punctures the body is located with an approximate error of $1 \mathrm{~mm}$. The base rotation $\theta$ is determined with a mean error of $2^{\circ}$ while the inclination angle $\Phi$ mean error is only $1^{\circ}$.

Phantom Experiments: Our first experiments on phantom took place at the Radiology Department of Grenoble's University Hospital. The CT Scanner used was a Siemens Somatom Volume Zoom. Our phantom is a foam rubber block (see fig. 4) in which we inserted a polyether-cetone disc with a $1 \mathrm{~cm}$ hole as a target. The experiment consists of trying to reach the target hole from an unknown position and orientation of the robot with the control of images coming from the imaging device, without any intervention of a manipulator and with a limited number of robot movement/localization loop. We tried 6 punctures ( 2 vertical and 4 with an arbitrary orientation) starting from different initial attitudes/positions. The target point, i.e. the exact pixel that was chosen on the screen, was reached in all the cases with an error smaller than $2 \mathrm{~mm}$ and the target disc hole was always reached. These results were obtained with a maximum of two image checking/robot movement loop including the initial localization. 


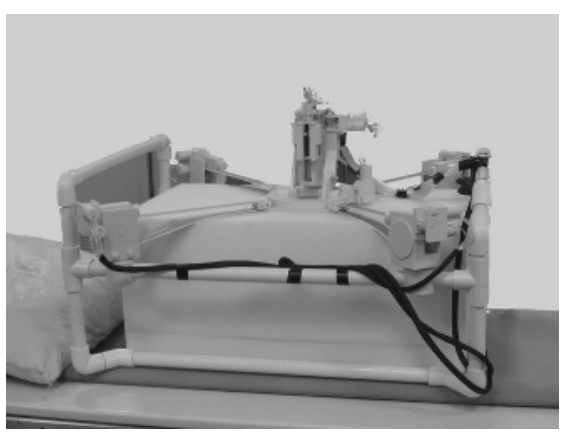

(a)

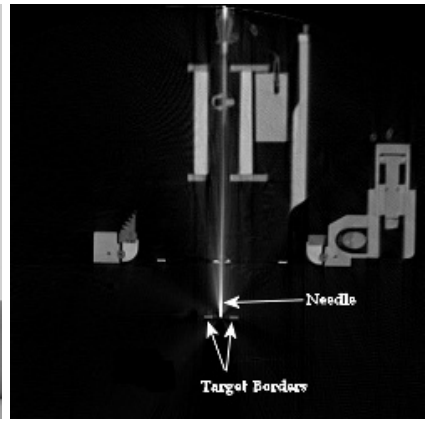

(b)

Fig. 4. (a) Robot on Phantom on scanner bed, (b) Vertical insertion result

\section{Discussion}

Open loop translation accuracy of the robot is satisfactory upon small distances (less than $30 \mathrm{~mm}$ ). Over this limit, the displacement is not as much accurate. Since the loop is closed by $\mathrm{CT} / \mathrm{MR}$ image acquisition, it is possible to remain very accurate, as the the final movement will be less than $30 \mathrm{~mm}$.

Some improvements on angle determination with images must be done in order to correctly determine LPR's orientation. A more complex image processing might improve the accuracy.

The experiments on phantom, even if their results are not statistically significant, are very promising. Our goal, which was reaching a $1 \mathrm{~cm}$ target without human intervention, is achieved in the particular experimental conditions described in sect. 3. It must now be proved that such results could be obtained in more difficult conditions i.e. on a non plane or moving phantom. In such conditions and in addition to problems caused by a non-plane geometry, needle deformations, which are not taken into account for the time being, might occur. In the case of a foam-rubber phantom, these are not significant. In real conditions, the needle curve might alter the precision of punctures.

One of the characteristics of LPR that has not yet been presented in this paper, is the possibility to release the needle from the clamp and grasp it again. This characteristic allows to let the needle follow the movements of patient's target organ while images are acquired, thus avoiding injuries. It also enables the robot to perform a deep insertion (when the target's distance from the entry point is greater than the needle-holder stroke) by releasing the needle and grasping it higher. This possibility has not been tested yet as our phantom is not mobile.

\section{Conclusion and Future Work}

A new light robot architecture for puncture is proposed in this paper. It is CT and MRI-compatible thanks to materials used to construct the robot and to its remote energy source. This robot uses pneumatic energy to perform translation, orientation and puncture. Experiments gave very promising results. 
Future work will concern clinical validation. In a near future, experiments will be done on a non-plane and mobile phantom in order to validate the prototype in almost real conditions. Animals experiments, and later human experiments, will follow as some improvements concerning the easiness of use of the robot will be carried out.

\section{References}

1. Masamune, K., Fichtinger, G., Deguet, A., Matsuka, D. and Taylor, R. H.,An Image Overlay System with Enhanced Reality for Percutaneous Therapy Performed inside CT Scanner, MICCAI'02, Tokyo, Japan, LNCS 2489, pp. 77-84, September 2002.

2. Gangi, A., Kastler, B., Arhan, J. M., Klinkert, A., Grampp, J.M., Dietemann, J.L., A compact laser beam guidance system for interventional CT, J Comput Assist Tomogr. 1994 Mar-Apr;18(2):326-8.

3. Susil, R. C., Anderson, J. H. and Taylor, R. H., A Single Image Registration Method for CT Guided Interventions, MICCAI'99,Cambridge, England, LNCS 1679, pp. 798-808, September 1999.

4. Stoianovici, D. Cadeddu, J. A., Demaree, R. D., Basile, H. A., Taylor, R. H., Whitcomb, L. L., Sharpe, W. N. Jr. and Kavoussi, L. R., An efficient needle injection technique and radiological guidance method for percutaneous procedures, CVRMedMRCAS'97, LNCS 1205, pp. 295-298, March 1997.

5. Masamune, K., Fichtinger, G., Patriciu, A., Susil, R., Taylor, R. H., Kavoussi, L. R., Anderson, J., Sakuma, I., Dohi, T. and Stoianovici, D., System for Robotically Assisted Percutaneous Procedures With Computed Tomography Guidance, Journal of Computer Assisted Surgery, Vol.6, No.6, pp.370-383, 2001.

6. Patriciu, A., Solomon, S., Kavoussi, L. and Stoianovici, D., Robotic Kidney and Spine Percutaneous Procedures Using a New Laser-Based CT Registration Method, MICCAI'01, The Netherlands, LNCS 2208, pp. 249-257, October 2001.

7. Kaiser, WA., Fisher, H., Vagner, J. and Selig, M. Robotic System for Biopsy and Therapy of Breast Lesions in a High-Field Whole-Body Magnetic Resonance Tomography Unit, Investigative Radiology, 35(8):513-519, August 2000.

8. Chinzei, K., Hata, N., Jolesz, F. and Kikinis, R., MR Compatible Surgical Assist Robot: System Integration and Preliminary Feasibility Study, LNCS 1935, MICCAI 2000, Oct 11-4, Pittsburgh, PA, pp. 921-30, 2000.

9. Fichtinger, G., Krieger, A., Susil, RC., Tanacs, A., Whitcomb, LL. and Atalar, E. Transrectal Prostate Biopsy Inside Closed MRI Scanner with Remote Actuation, under Real-Time Image Guidance. MICCAI'02, Tokyo, Japan, LNCS 2488, Part 1, pp 91-98, Springer Verlag, September 2002

10. Susil, RC., Krieger, A., Derbyshire, JA., Tanacs, A., Whitcomb, LL., Fichtinger, G. and Atalar, E., System for MR Image-Guided Prostate Interventions: Canine Study Radiology 2003; 228: 886-894. 